\title{
DEDDNTE

\section{1) Aplicação da avaliação geriátrica ampla em paciente idosa internada: um relato de caso}

\author{
Vanessa Teles Felinto Mello'
}

\section{Resumo}

A população brasileira vem passando por um processo de envelhecimento, com aumento da presença da síndrome da fragilidade, sendo importante a adoção de métodos de triagem e avaliação que identifiquem idosos em risco. Uma avaliação que se mostra eficiente em dar uma visão global e completa da situação de saúde do idoso é a Avaliação Geriátrica Ampla (AGA), que é um instrumento de avaliação multidimensional, multidisciplinar, que avalia a capacidade funcional, saúde física, cognitiva e mental do idoso, além das circunstâncias socioambientais.

Palavras-chave: Fragilidade. Avaliação geriátrica ampla. Capacidade funcional.

\section{Introdução}

Com o envelhecimento da população brasileira, percebe-se um aumento da síndrome da fragilidade, exigindo o uso de diferentes e múltiplos métodos de triagem e avaliação para identificar quais são os idosos que estão em risco. Uma possível avaliação que tem se mostrado eficiente é a Avaliação Geriátrica Ampla (AGA).

\section{Metodologia}

Relato de caso clínico realizado com paciente do sexo feminino, 78 anos, feoderma, do lar, divorciada, alfabetizada. Internada na Clínica Cirúrgica do Hospital Regional da Ceilândia, Distrito Federal, para cirurgia ginecológica de baixo risco (histerectomia). A avaliação da paciente foi realizada um dia após o pós cirúrgico. Para a realização do relato de caso clínico foram utilizados os dados exigidos pelo instrumento de avaliação. Objetivo: aplicar a AGA em uma idosa internada em hospital, de forma a demonstrar sua importância para a avaliação dessa, bem como na definição de um plano terapêutico mais adequado.

Nutricionista e Mestre em Saúde do Idoso, pela Fundação de Ensino e Pesquisa em Ciências da Saúde do Distrito Federal (Fepecs). Endereço para correspondência: SMHN Quadra 03, conjunto A, Bloco 1 Edifício Fepecs. 70.710907. Brasília, DF, Brasil. Email: vanessatfelinto@gmail.com 


\title{
Resultados e Conclusão
}

A paciente em questão mostra-se muito ativa e sem necessidades especiais quando avaliada de maneira superficial. A partir da aplicação da AGA pode-se identificar alterações nas funções de mobilidade e cognição que talvez não seriam identificadas se esse instrumento não tivesse sido utilizado. Contudo, em relação à cognição, devemos considerar o fato de a paciente ser somente alfabetizada o que influencia diretamente nesse parâmetro. A AGA se mostra como uma ferramenta que permite a identificação e avaliação do estado global do paciente idoso, resultando num plano de tratamento mais eficaz e que pode ser traçado não só na presença da doença, mas também para a manutenção e recuperação da capacidade funcional desse idoso. Por ser de utilização interdisciplinar e de fácil aplicação.

\section{Application of the broad geriatric assessment in hospitalized elderly patients: a case report}

\begin{abstract}
The Brazilian population is undergoing an aging process, with an increased presence of fragility syndrome, being important the adoption of screening and evaluation methods that identify elderly at risk. An evaluation that is efficient in giving a global and complete view of the health situation of the elderly is the Comprehensive Geriatric Assessment (AGA), which is a multidimensional multidimensional assessment tool that assesses functional capacity, physical, cognitive and mental health of the elderly, in addition to socio-environmental circumstances.
\end{abstract}

Keywords: Fragility. Comprehensive geriatric assessment. Functional capacity. 